Bundesgesundheitsbl 2012 $\cdot 55: 753-755$

DOI 10.1007/s00103-012-1501-8

Online publiziert: 7. Juni 2012

(c) Springer-Verlag 2012

\section{Pigeot · W. Ahrens}

BIPS - Institut für Epidemiologie und Präventionsforschung, Bremen

\title{
Quo vadis, Kohorte?
}

Liebe Leser und Leserinnen,

das vorliegende Heft beschreibt in zahlreichen Beiträgen die methodischen Ansätze und das wissenschaftliche Potenzial prospektiv angelegter Kohortenstudien für die epidemiologische Gesundheitsforschung. Dabei gliedert es sich grob in zwei Teile: Die Beiträge des ersten Teils beschreiben Studien bei Erwachsenen und die aus ihnen gewonnenen Erkenntnisse, während sich die Beiträge im zweiten Teil auf Kohorten mit Kindern und Jugendlichen beziehen.

Die in diesem Heft dargestellten Kohortenstudien sind längsschnittliche Beobachtungen von Bevölkerungsgruppen, bei denen über einen definierten Zeitraum hinweg ausgehend von einer sogenannten Basisuntersuchung ausgewählte Bevölkerungsgruppen bezüglich gesundheitlicher Endpunkte nachverfolgt werden, um gesundheitserhaltende und krankheitsverursachende Faktoren zu identifizieren. Unter den beobachtenden epidemiologischen Studiendesigns liefern prospektive Kohortenstudien den höchstmöglichen Evidenzgrad für Kausalbeziehungen, weil nur sie die zeitliche Abfolge zwischen Exposition gegenüber einem oder mehreren Risikofaktoren und einer Erkrankung eindeutig nachbilden können.

Die im Titel des Editorials gestellte Frage hat eine doppelte Bedeutung: Sie betrifft zum einen die gesundheitlichen Endpunkte, auf die eine Kohortenstudie ausgerichtet ist, das heißt die interessierenden Krankheiten, die in ihrer Entwicklung prospektiv erforscht werden sollen. Zum anderen geht es um die Frage, wie in Zukunft Kohortenstudien geplant und ausgestaltet werden müssen, um aktuellen Fragestellungen und den sich daraus ergebenden Anforderungen gerecht werden zu können. Eine neue Kohorte sollte anschlussfähig sein, um in einer vorangegangenen Studie beobachtete Zusammenhänge replizieren zu können, sie muss aber gleichzeitig so angelegt sein, dass hinsichtlich bislang nicht untersuchter Expositionen, biologischer Marker oder Endpunkte ein zusätzlicher Erkenntnisgewinn entsteht.

Im angelsächsischen Raum ist die Bedeutung großer Kohortenstudien bereits seit Langem anerkannt. So wundert es nicht, dass ein Meilenstein für moderne Prospektivstudien in Amerika gelegt wurde: 1948 begann die immer noch andauernde Framingham Heart Study, benannt nach der amerikanischen Kleinstadt Framingham, deren Bürger und mittlerweile auch ihre Nachkommen in diese Kohorte eingeschlossen wurden mit dem Ziel, die Ätiologie von HerzKreislauf-Erkrankungen zu erforschen. Damit wurde die Framingham Heart Study zum Vorbild für epidemiologische Kohortenstudien, und viele ihrer Designelemente fanden Eingang in nachfolgende Studien. Viele ausländische und internationale Kohorten wurden seitdem mit unterschiedlichen Endpunkten aufgesetzt. Einen Überblick über große internationale Kohorten und deren Bedeutung für die epidemiologische Forschung gibt der einleitende Beitrag von Ahrens und Pigeot in diesem Heft.

In Deutschland werden neben zahlreichen regional begrenzten Kohortenstudien mit unterschiedlichsten Endpunkten, von denen die größten in diesem Heft vorgestellt werden, bislang zwei bundesweite Kohorten gepflegt: (1) das sozio-oekonomische Panel (SOEP), dessen erste Erhebungswelle 1984 in Westdeutschland und 1990 in Ostdeutschland stattfand und in dem jährlich mehr als 20.000 Bundesbürger befragt werden.
Die im SOEP erhobenen Daten beziehen sich unter anderem auf allgemeine $\mathrm{Ge}$ sundheitsindikatoren, die insbesondere über Fragen gesundheitlicher Ungleichheit und von Health Economics Aufschluss geben können, wie Schupp in seinem Beitrag ausführt; (2) die „Studie zur Gesundheit Erwachsener in Deutschland" (DEGS), die vom Robert Koch-Institut im Rahmen des Gesundheitsmonitorings in einer ersten Welle von 2008 bis 2011 durchgeführt wurde und an den Bundesgesundheitssurvey von 1998 anschließt. Von den 8154 Personen, die an dem DEGS teilgenommen haben, wurden Daten erhoben, mit denen sich zeitliche Trends in der Entwicklung der gesundheitlichen Lage in Deutschland und die gesundheitliche Entwicklung im Lebensverlauf beurteilen lassen. Eine kurze Beschreibung des DEGS findet sich im Beitrag von Gößwald und Koautoren.

Trotz ihrer umfangreichen Datensammlung sind diese beiden Kohortenstudien nicht auf die Erforschung der Ätiologie von Erkrankungen ausgerichtet. Dies leisten eher die in den verschiedenen Beiträgen beschriebenen regionalen Kohorten, die jedoch den Nachteil haben, keine bundesweit vergleichbaren Daten zu liefern, die anhand eines einheitlichen Studienprotokolls und unter Einsatz derselben Befragungs- und Messinstrumente erhoben wurden. Diese Lücke in der deutschen Gesundheitsforschung soll zukünftig mit der Durchführung einer nationalen Kohortenstudie geschlossen werden. Geplant ist die Untersuchung von 200.000 Bundesbürgern im Alter von 20 bis 69 Jahren mit dem Ziel, Risikofaktoren, Verlauf, subklinische Vorstufen und auftretende funktionelle Veränderungen von bzw. bei chronischen Erkrankungen des HerzKreislauf-Systems und der Lunge, Dia- 
betes, Krebs sowie neurodegenerativen/psychiatrischen Krankheiten und Infektionskrankheiten zu ermitteln. Der Beitrag von Wichmann und Koautoren zeigt die Bedeutung der Nationalen Kohorte für die epidemiologische Forschung in Deutschland und ihr Innovationspotenzial insbesondere im Hinblick auf den Aufbau einer neuen Kohorte auf.

Wie bereits oben angesprochen, existiert in Deutschland eine Reihe weiterer regionaler Kohortenstudien, von denen in diesem Heft die bedeutendsten vorgestellt werden. So beschreibt Völzke in seinem Beitrag die in Nordostdeutschland seit 1997 angesiedelte „Study of Health in Pomerania" (SHIP), die auf der einen Seite die Schätzung der Prävalenz und Inzidenz häufiger Risikofaktoren und Erkrankungen und auf der anderen Seite die Erforschung der Ätiologie von manifesten Erkrankungen zum Ziel hat. Zu diesem Zweck wurden initial 4308 Personen in die Studie eingeschlossen und zahlreichen Untersuchungen, wie zum Beispiel somatometrischen und Blutdruckmessungen, Zahn-, Haut-, kardiometabolischen und verschiedenen Ultraschalluntersuchungen bis hin zum Ganzkörper-MRT, unterzogen. Ebenfalls in Ostdeutschland, und zwar in Halle an der Saale, wird seit 2002 die CARLA-Studie („Cardiovascular Disease, Living and Ageing in Halle") durchgeführt, die im Beitrag von Haerting und Koautoren näher beschrieben wird. In dem Baseline-Survey wurden 1779 Personen im Alter von 45 bis 83 Jahren speziell mit Blick auf Risikofaktoren für Herz-Kreislauf-Krankheiten, insbesondere für eine eingeschränkte Herzfrequenzvariabilität als Indikator für eine autonome Dysfunktion untersucht. Eine weitere Studie in Ostdeutschland wird von Heinrich und Wölke vorgestellt, und zwar die Erfurter Männer-Kohorte-Studie (ERFORTStudie), die bereits 1973 begann und von 1973 bis 1975 erstmalig 1160 Männer im Alter von 35 bis 61 Jahre mit dem Hauptziel einschloss, kardiovaskuläre Risikofaktoren, lebensstilabhängige Verhaltensweisen und psychosoziale Charakteristika im Hinblick auf die gesamt- und todesursachenspezifische Mortalität und Morbidität zu untersuchen.
Die Heinz Nixdorf Recall Studie, vorgestellt in dem Beitrag von Erbel und Koautoren, hat von Januar 2000 bis August 2003 im Ruhrgebiet Daten an 4814 Personen im Alter von 45 bis 75 Jahren erhoben, um zu einer verbesserten Vorhersage kardiovaskulärer Ereignisse, insbesondere von Myokardinfarkt und plötzlichem Herztod, unter Einsatz moderner bildgebender und nicht bildgebender Verfahren zu gelangen. Zwei weitere in Nordrhein-Westfalen angesiedelte Kohorten werden von Berger und Wersching in zwei Beiträgen vorgestellt. Es handelt sich dabei zum einen um die Dortmunder Gesundheitsstudie (DOGS), die seit 2003/4 in einer Kohorte von 2291 Personen die Prävalenz und Inzidenz verschiedener Kopfschmerzarten und anderer chronischer Erkrankungen sowie deren Einfluss auf den Lebensalltag betroffener Personen ermittelt, und zum anderen um eine neuere, seit 2009 bestehende Kohorte, die sogenannte BiDirect-Studie, in Münster, die den Zusammenhang zwischen Depressionen und kardiovaskulären Erkrankungen untersucht.

In Mainz wird seit 2007 die Gutenberg Gesundheitsstudie durchgeführt mit dem Ziel, die Risikovorhersage für HerzKreislauf-, Krebs- und Augenerkrankungen, metabolische Erkrankungen sowie Erkrankungen der Psyche und des Immunsystems zu verbessern. Die Basisuntersuchung schloss 15.000 Personen im Alter von 35 bis 74 Jahren in ein fünfstündiges Untersuchungsprogramm ein. Eine ausführlichere Beschreibung geben Wild und Koautoren in ihrem Beitrag. Wie von Nöthlings und Krawczak in ihrem Beitrag ausgeführt, wurde die in Kiel beheimatete Biobank PopGen 2003 zur Erforschung genetischer Risikofaktoren für komplexe Erkrankungen eingerichtet und zwischen 2005 und 2007 zu einer Kohorte ausgebaut, um die zeitlichen Veränderungen bei entzündungsbezogenen Biomarkern zu untersuchen.

Kohorten, in die Kinder eingeschlossen werden, sind für die Erforschung von Erkrankungen in der Lebensperspektive besonders wertvoll, wobei ein großes Innovationspotenzial von Geburtskohorten zu erwarten ist, da nur mit ihnen die Bedeutung von intrauterinen und früh- kindlichen Faktoren für die spätere Entwicklung von Gesundheitsstörungen in vollem Umfang untersucht werden kann.

Vergleichbar zur DEGS wird vom Robert Koch-Institut die Studie zur Gesundheit von Kindern und Jugendlichen in Deutschland (KIGGS) durchführt. Dazu wurden von 2003 bis 2007 in 167 Städten und Gemeinden 17.641 Kinder und Jugendliche im Alter von 0 bis 17 Jahren und ihre Eltern in eine Basisuntersuchung eingeschlossen, in der wesentliche Indikatoren der körperlichen, psychischen und sozialen Gesundheit erhoben wurden. Wie in dem Beitrag von Hölling und Koautoren ausgeführt, sollen unter anderem Aussagen über Trends in der Prävalenz von Übergewicht und Adipositas, der Inzidenz von atopischen Erkrankungen und der Persistenz bzw. Remission von psychischen Auffälligkeiten und Störungen gewonnen werden. Das Deutsche Kinderkrebsregister stellt eine einmalige Basis für die Langzeitnachbeobachtung von Krebserkrankungen im Kindesalter dar. In die Kohorte werden Kinder eingeschlossen, deren Krebserkrankung vor dem 15. Geburtstag aufgetreten ist. Die Kohorte, die von Kaatsch und Grabow beschrieben wird, besteht aus mehr als 25.000 Patienten.

Vergleichbar mit der Idee der Nationalen Kohorte wird zurzeit in Deutschland auch die Konzeption einer bundesweiten umweltepidemiologischen Geburtskohorte diskutiert. Schmidt und Koautoren stellen das Rahmenkonzept in ihrem Beitrag vor, nach dem 100.000 bis 200.000 Eltern möglichst früh in der Schwangerschaft rekrutiert werden sollen, um insbesondere die folgenden Gesundheitsthemen $\mathrm{zu}$ erforschen: reproduktive Entwicklung, geburts- und schwangerschaftsbezogene Ereignisse, Asthma, Allergien und Atemwegserkrankungen, Adipositas, Insulinresistenz und Diabetes.

Diese Themen werden zum Teil bereits in kleineren, regional begrenzten Kinderkohorten erforscht. So beschreiben Pohlabeln und Koautoren eine Geburtskohorte in Nordwestdeutschland, bei der Ende der 1990er-Jahre mehr als 3000 Neugeborene rekrutiert wurden, um unter anderem den Zusammenhang 
von Stilldauer bzw. Haustierhaltung und dem Auftreten von Allergiesymptomen $\mathrm{zu}$ untersuchen. Zwei weitere Geburtskohorten GINIplus und LISAplus werden von Heinrich und Koautoren vorgestellt. Beide Kohorten beschreiben primär den natürlichen Verlauf chronischer Erkrankungen im Kindesalter sowie intermediäre Phänotypen und Funktionseinschränkungen, analysieren deren Determinanten und identifizieren mögliche Effektmodifikationen durch genetische Polymorphismen. Die Untersuchungen begannen Mitte der 1990er-Jahre und schlossen 5991 (GINIplus) bzw. 3097 (LISAplus) Neugeborene ein. In Dortmund werden seit 1984 jährlich ca. 40 Neugeborene in die DONALD-Studie („Dortmund Nutritional and Anthropometric Longitudinally Designed Study") eingeschlossen, um komplexe Wechselwirkungen zwischen Ernährung, Stoffwechsel, Entwicklung und Wachstum zu analysieren. Die Ergebnisse werden von Buyken und Koautoren in ihrem Beitrag diskutiert.

Die Erforschung der kindlichen Adipositas und deren Prävention ist Thema zweier Kohorten. Die Kieler Adipositas-Präventionsstudie (KOPS) wird seit 1996 in Kiel durchgeführt und schloss 4997 Schüler in eine Eingangsuntersuchung ein. Die hier von PlachtaDanielzik und Koautoren präsentierten Ergebnisse fokussieren unter anderem auf den optimalen Zeitpunkt und geeignete Ansätze zur Adipositasprävention. Die von Hebestreit und Ahrens vorgestellte IDEFICS-Studie („Identifcation and Prevention of Dietary- and LifestyleInduced Health Effects in Children und Infants") untersucht nicht nur die Ursachen kindlicher Adipositas, sondern allgemein von ernährungs- und lebensstilbedingten Erkrankungen. Darüber hinaus werden Strategien zur Primärprävention derartiger Erkrankungen entwickelt. Dazu wurden in Deutschland als Teil eines europäischen Konsortiums (siehe dazu auch den Beitrag von Ahrens und Pigeot) von September 2007 bis Mai 20082065 Kinder im Alter von 2 bis 9 Jahren einem umfangreichen Untersuchungsprogramm unterzogen, das in den Jahren 2009 bis 2010 wiederholt wurde.
In der Hoffnung, dass Ihnen die Beiträge die Bedeutung epidemiologischer Kohortenstudien verdeutlichen werden, verbleiben wir

Ihre

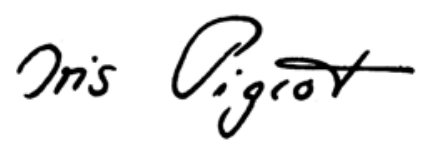

Iris Pigeot

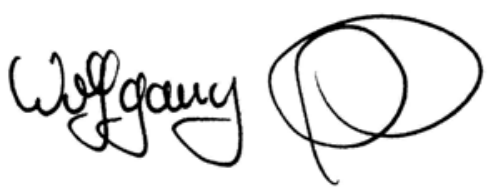

Ihr Wolfgang Ahrens

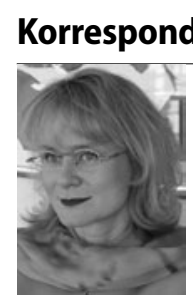

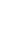

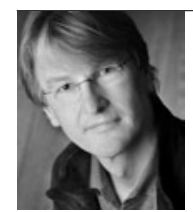

Prof. Dr. I. Pigeot

BIPS - Institut für Epidemiologie und Präventionsforschung Achterstr. 30, 28359 Bremen pigeot@bips.uni-bremen.de

Prof. Dr. W. Ahrens

BIPS - Institut für Epidemiologie und Präventionsforschung Achterstr. 30, 28359 Bremen ahrens@bips.uni-bremen.de 